\title{
Studying the Role of Cognition in Depression: Well-Trodden Paths and Cul-de-Sacs
}

\author{
James C. Coyne ${ }^{1}$ \\ University of Michigan \\ Ian Gotlib \\ University of Western Ontario
}

In their commentary on our earlier review (Coyne \& Gotlib, 1983), Segal and Shaw minimize the problems faced by the cognit, e approach to the study of depression. Their repeated references to cognitive processes that are latent and therefore not reported by subjects represent a substantial retreat from empiricism. In general, the notion of schema or schematic processing remains problematic. Published research does not support the existence of an identifiable cognitive vulnerability to depression. Other conceptual, methodological, and empirical difficulties are noted. We argue that the central defect in current cognitive approaches to depression is their inattention to the difficulties faced by depressed persons in their everyday environments, how they cope, and with what consequences.

KEY WORDS: depression; schema; cognition.

Segal and Shaw present a discussion intended to serve two purposes: first, to clarify the misconceptions they feel were present in our earlier review of cognitive approaches to the study of depression (Coyne \& Gotlib, 1983), and second, to offer an updated perspective on the issues discussed in that article. In this paper we will address these two aspects of Segal and Shaw's article, and in addition, we will take this opportunity to present briefly our own updated view of the role of cognition in depression.

'Address all correspondence to James C. Coyne, University of Michigan Medical School, Ann Arbor, Michigan 48109. 
In our original paper, we acknowledged that it was the outstanding achievement of the cognitive perspective that such a large literature was available for critical scrutiny. Overall, however, we found that the empirical support for the most distinctive claims of the two major cognitive models was less consistent than had generally been assumed. Furthermore, we found that key concepts were not well specified, thinly veiled tautologies were rampant, and weak correlational methodologies were being accepted as the basis for strong causal inferences. Our concluding assessment was not just that the cognitive models were being embarrassed by the accumulating data, but that beyond this, there was cause for pessimism, in that any data that could conceivably be generated by the methods currently in use were limited in what they could offer for the models' refinement.

Some of the issues were raised in that article can be more easily resolved than can others. We remain conceived that how depressed people think will prove important in any comprehensive model of this disorder. We are also convinced, however, that current cognitive models face some formidable conceptual, methodological, and empirical difficulties, and that the field would benefit from the generation of rival hypotheses and alternative methodologies. A viable model of the role of cognition in depression will likely look quite different than what is currently available, and radically different methodologies are needed if the claims of such a model are to be empirically grounded.

Segal and Shaw indicate that they accept some of our earlier conclusions regarding conceptual and methodological problems of the cognitive perspective, although unfortunately they do not elaborate on which conclusions there is agreement or on what the implications of these conclusions are for the claims of the cognitive perspective. Beyond that, Segal and Shaw suggest that we have obliquely portrayed some pivotal issues in the study of the role of cognition in depression, and that this presentation diminishes the validity of our conclusions. We are confident that a rereading of our original review will answer many of their concerns. Other of their points involve major revisions of the original claims of the cognitive perspective. Not all of these revisions are acknowledged as such, however, and we believe that some of their suggestions direct researchers down already well-trodden paths and cul-de-sacs that are not likely to yield any surprising discoveries.

Our general sense is that Segal and Shaw have minimized the problems faced by the cognitive perspective and have overestimated the potential of some solutions that are currently being attempted. Furthermore, the results of many of the new studies that they cite in their update of empirical research only strengthen our original conclusions. In addition to replying to their article, therefore, we will delineate further the challenges that we feel face both current cognitive models of depression and whatever alternatives may succeed them. 


\section{POTATOES OR SPUDS?}

Segal and Shaw wish to prevent difficulties that they perceive as limited to the learned helplessness model from reflecting badly upon Beck's model, and they caution that they "regard it as premature to view these two models as identical." However, the only situation in which they are able to contrast the two models involves expectancy changes following laboratory failure experiences. This is an unfortunate choice of experimental paradigms. As we noted in our original review, interest in such studies waned with an accumulation of null findings and the recognition that even positive findings would be ambiguous in their interpretation. Furthermore, Abramson, Seligman, and Teasdale (1978) themselves dismissed the relevance of such studies to their reformulation: "In the absence of knowledge about individual attributions, the reformulated model cannot make clearcut predictions about expectancy changes and helplessness" (p. 63).

Ultimately, existing cognitive models of depression may be refined in a way that allows the execution of decisive empirical tests and the emergence of a single dominant paradigm. However, we believe that the current stalemate is indicative of some basic conceptual problems: Cognitive concepts are so slippery and indeterminate that clashes between a given model and data are likely to be unsatisfactory in their outcome.

Key concepts in the study of cognition in depression are probably best seen as loosely metaphorical in nature. Cognitive researchers have rarely been able to go beyond simple demonstrations that depressed persons say negative and pessimistic things about themeselves and their situations. They have yet to begin to establish the basis for their strong claims about how these selfreports came about. As we noted in our earlier review, current methodologies do not allow unambiguous demonstrations that depressed persons are any more distorted in their thinking than nondepressed persons. Such demonstrations may not even be attainable. The penchant for elaborate models of information processing is therefore not matched by the existence of methodologies that allow much discrimination among rival descriptions of these processes. Segal and Shaw exemplify a long-standing gap between method and inference in their uncritical acceptance of what appears to be a convention measure of attitudes, the Dysfunctional Attitude Scale (DAS; Weissman \& Beck, 1978), as evidence of the "operation of hyperactive schemata." Furthermore, the increasing reference to latent and deep processes by Segal and Shaw and others (Beck, 1984; Riskind \& Rholes, 1984) is reducing the possible points of contact between cognitive formulations and empirical data. As Silverman, Silverman, and Eardley (1984b) state, for cognitive theorists to fall back to a position that in depression-prone people dysfunctional attitudes are not conscious but latent (i.e., they are there but not detectable) seems "an undignified retreat." 


\section{PROBLEMS WITH SCHEMA-BASED EXPLANATIONS OF DEPRESSION}

Segal and Shaw seem particularly concerned that the difficulties of the learned helplessness model will damage the credibility of Beck's schema-based model of depressive thinking, but they fail to come to terms with the pitfalls of explanations based on this concept. The concept of schema is typically used in discussions of depression in a way that is quite different from its original usage in experimental cognitive psychology, and it now provides a mere label rather than much explanatory power. One might recall Moliere's doctoral candidate explaining that the "cause and reason" that opium puts people to sleep is that it possesses a dormitive principle.

Depressogenic schemata are taken as an explanation of why depressed persons persist in self-defeating and painful attitudes in the face of ostensibly contradictory experiences. Yet, empirically at least, the evidence that is offered for the operation of schema is generally limited to the very negative verbalizations that the concept explains: Depressed persons make negative verbalizations because of the operation of hyperactive negative schemata, and we know this because they make negative verbalizations.

Furthermore, Shaw (1979) himself has cogently noted, commitment to schema as an explanation makes it difficult to accommodate the observation that humans (including depressed persons) can readily make changes in their thought processes as a result of their experience (see Beck, 1967, for early findings concerning depressed persons' response to success experiences). Finally, to argue, as cognitive theorists do, that perception is schematici.e., that involves an application of stable, organized representation of past experiences in order to structure new ones-introduces a host of new problems. For one, it begs the question of what guides the activation and matching of schemata to stimuli. If one attempts to treat schematic explanations as more than loose metaphors, one quickly becomes lost in logical difficulties, including an infinite regress (Heil, 1983).

We noted in our original article that, from an empirical perspective, if one fails to find expected differences between depressed and nondepressed persons in reactions to a stimulus, it is embarrassing to Beck's model. Yet if one obtains hypothesized results, one is still left far from having strong support for the operation of the specific processes posited by the model. Segal and Shaw convey little sense of how one should proceed in order to obtain evidence that these processes, rather than some alternative, are responsible for the differences that are observed. Such difficulties are a matter of fuzzy thinking, not the lack of empirical data, and they are not going to be readily resolved. 


\section{CAUSALITY}

Segal and Shaw state that we were correct in pointing out that the etiological stance in "earlier formulations" of the cognitive theory was one of "cognitive primacy." They go on to state that we did not concede in our review that "Beck's model has evolved in its acknowledgment of the complexity of causal factors in depression." In support of this evolution, Segal and Shaw cite a number of papers, all of which were published after our review had gone to press. It thus appears that we took a reasonable stance in working with the then current versions of the cognitive models.

It may now be appropriate to bow to the accumulation of empirical findings and acknowledge that the evidence that cognitions are causal in depression is weak. However, in the interest of good scholarship, one should also admit that such claims of causality have been frequently made in the past and continue to be made, and that-despite assertions by Segal and Shaw - the "search for a fundamental causal priority of cognition over affect" is not merely the invention of critics of the models. Numerous examples of causal statements could be cited. For instance, authors who have worked closely with both Beck and Shaw have written: "The theory makes a clear causal relationship between cognition and behavioral, affective, and physiological disturbances. The theory goes beyond descriptive detailing of the experience of depression and asserts that cognitions cause and maintain the depressive syndrome" (Rush \& Giles, 1982, p. 151). Furthermore, Beck himself has very recently stated that "the cognitive theory of depression proposes that depression is primarily a result of the tendency to view the self, the future, and the world in an unrealistically negative manner" (Sacco \& Beck, 1985, p. 4). In fact, Sacco and Beck follow this statement with a review of those studies "which provide support for the notion that negative perceptions are causally related to the other symptoms of depression" (p. 21). Thus, it is still not clear that the current cognitive model is significantly different from the formulation described in our review.

It would be useful to explore briefly what is at stake with the causal claims of the cognitive models. That depressed persons are pessimistic and complain about themselves was noted as early as the writing of Robert Burton in the 17th century and even the Book of Job. This observation was not left for the cognitive theorists to discover; rather, they suggested a new and special significance to these complaints. They argued that the study of depressed persons' self-complaints and negativity is not merely a descriptive exercise but an exploration of the cause of depression.

There are obviously a number of alternatives to the cognitive theorists' strong claims about causality. First, one might suggest, as Klein (1974) has 
about some types of depression, that depressives' complaints reflect a secondary demoralization and self-blame for their condition, and that these complaints are best seen as an effect rather than a cause of depression. Second, one might argue that it is simply tautological that depressed persons complain about themselves and their situations: Not surprisingly, people who are chosen because they endorse self-complaints on the BDI, for instance, are likely to endorse self-complaints when they are given other possibilities to do so. There are other interpretations possible (we will outline one in particular later in this paper), but these two are important as those to which proponents of the cognitive models have had to react. Thus, the risk has always been that purported measures of cognitions associated with depression will be dismissed either as the effects of depression or as relatively uninteresting variations on some of the content of conventional measures of depression. Invoking causal arguments has been a defense of the usefulness of such measures.

Segal and Shaw now reject the idea that thinking cause depression. The task then remains of establishing why the study of depressive cognitions remains interesting. Segal and Shaw indicate that one key reason is that it may be possible to identify a cognitive vulnerability to depression. This echoes a previous suggestion of Kovacs and Beck (1978): "Certain cognitive processes seem chronically atypical among depressed patients and may represent a stable characteristic of their personality" (p. 530). One may note in passing that the idea of a cognitive vulnerability is a causal one. Restated, the vulnerability hypothesis is that an identifiable, preexisting cognitive characteristic of some people will cause them to become depressed when they are confronted with a particular set of circumstances. However, the important point is that there is an empirically testable hypothesis here, even if there are more complications than Segal and Shaw acknowledge.

\section{VULNERABILITY MARKERS, PRODROMAL SIGNS, AND RESIDUALS}

We are puzzled by Segal and Shaw's depiction of negative results of Lewinsohn, Steinmetz, Larsen, and Franklin (1981) as "more an example of poor formulation than they are a refutation of the cognitive model.... By regarding dysfunctional thinking in an undifferentiated way the assessment employed by Lewinsohn et al. (1981) failed to test the predictions derived from the cognitive model." Lewinsohn et al. obtained self-report and interview data from 998 community residents at two times, approximately 8 months apart. Measures of a number of distinct constructs were obtained: causal attributions, expectancies of positive and negative outcomes (which constitute part of Beck's triad), irrational beliefs, perception of control, and self-esteem. Diagnoses were obtained on the basis of structured interviews. 
Contrary to the hypotheses that might have been generated by the cognitive theories, none of these measures was found to predict the onset of depression. Those persons who became depressed at Time 2 were not characterized premorbidly by pessimism, misattributions, and irrational negative beliefs. Lewinsohn et al. did not specifically test for the interaction between cognitive vulnerability and life stress. However, given their large $N$, one could expect that if such interaction were significant, it would be readily detectable as a main effect for the cognitive measures - except in the unlikely case that there was a strong negative correlation between the cognitive measures and life stress. The absence of the main effect indicates the likely absence of an untested interaction effect. (This point is widely misunderstood in the literature concerning cognitive vulnerability for depression. See Metalsky, Abramson, Seligman, Semmel, \& Peterson, 1982, as an example of this misunderstanding.)

This study has important implications for claims that there exists an identifiable cognitive vulnerability to depression. Segal and Shaw's attempt to escape its implications is by restricting the concept of vulnerability to persons who have been depressed in the past. This is a substantial revision of the concept, and it begs some important questions. For example, how does a person's first major depression come about, and what is the role of cognition in that process? Are purported cognitive features of depression best seen as effects of the disorder that accumulate with the passage of time spent depressed? Is cognitive vulnerability acquired by being clinically depressed?

Intriguing though these theoretical questions may be, their interest is diminished by the failure of efforts to identify cognitive differences enduring beyond a thorough recovery from a depressive episode. Segal and Shaw review studies that have used the DAS. They cite a number of recent studies, the published ones of which do not support their claims regarding the theoretical or practical utility of this measure.

Four of the relevant published studies cited by Segal and Shaw (Hamilton \& Abramson, 1983; Reda, 1984; Silverman, Silverman, \& Eardley, 1984a; Simons, Garfield, \& Murphy, 1984) found that DAS scores of depressed patients returned to normal levels after the depressive episode was judged to have remitted. Segal and Shaw, however, seem to have a preference for some unpublished studies over these. These studies suggest that although DAS scores may be lower at the end of the episode, they do not return to the normal range. While they were not available to us, Segal and Shaw note that the two unpublished studies that support the existence of a cognitive vulnerability marker involved assessments only 2 to 3 weeks after judgment of remission. As Silverman et al. (1984b) note, the "maladaptive attitudes gap" between treated depressives and normal controls may be a function of the incomplete recovery of the depressed patients. The note that Eves and Rush (1984), for example, used a Hamilton score of 10 or less a criterion 
of recovery, and they suggest that different results may have been obtained had more stringent criteria been used.

Some cognitive theorists, as we have noted, are nonplussed by these DAS findings. Riskind and Steer (1984), at Beck's Center for Cognitive Therapy, for example, discuss the Silverman et al. (1984a) findings that the DAS scores of depressed patients were higher during the patients' depressive phase than during remission, and actually suggest that these findings are consistent with Beck's theory. They cite a quote from Beck arguing that "even though these negative attitudes... may not be prominent or even discernible at a given time, they persist in a latent state... Once activated, these concepts dominate the person's thinking and lead to the typical depressive symptomatology." (Note the causal nature of this statement.) Riskind and Steer conclude that "therefore, the decrease in dysfunctional attitudes that Silverman et al. found is exactly what the cognitive theory would predict" (p. 1111). We find ourselves disappointed in their reasoning, and as we stated in our earlier article, "it is likely to be difficult to distinguish empirically a hypothesis concerning latent cognitive factors from the traditional assumption that when confronted with certain internal and external stimuli, some people become depressed, and negative thinking is simply one characteristic of this state" (Coyne \& Gotlib, 1983, p. 499).

Other cognitive theorists have recognized that the findings with the DAS have been damaging to the cognitive models. Rush and Giles (1982), for example, cite an unpublished study in which they found that more severe depressive symptomatology was associated with greater endorsement of DAS items in both unipolar and bipolar patients, as compared to those patients who were less symptomatic or remitted. Rush and Giles interpreted the relationships that they found between depression and this purported measure of schemas as being not as clear-cut as predicted by the cognitive theory. Nearly half of the symptomatic patients did not endorse any more dysfunctional attitudes than did normal college students without measured psychopathology. "Either the DAS is an inadequate instrument to measure schemas, or schemas play a key role in only a subgroup of depressed patients. Further research into the role and relevance of schemas is needed.... The role of schemas posited by cognitive theory has received little confirmatory empirical support" (p. 174).

It is possible to argue for the abandonment of the DAS rather than the hypotheses that its use has failed to support. Yet, given that the selfcomplaint of the type tapped by measures of purported depressive cognition also happen to be symptoms of depression, it will be difficult to establish that any such measure is a valid and reliable cognitive marker for depression and also not state-dependent. For example, certain features or symptoms of depression may be prodromal (Williams, 1985) rather than vulnerability 
factors, and close observation of depressed persons may demonstrate that these symptoms are early indications of a progressive state of affairs that was set in motion by other factors. As an analogy, sniffles are better viewed as a prodromal symptom of a common cold than as a vulnerability factor. Alternatively, because criteria for demarcating the end of an episode of depression are arbitrary, certain features such as elevated DAS scores may merely represent residual symptoms that would disappear over a longer period of time. Finally, even if such features were found to persist after other symptoms of the depression had subsided, it is still possible that they may reflect involvement in an intractible situation such as an unsupportive marriage that preceded both the onset of the depression and the appearance of these particular features.

\section{WHAT EVER HAPPENED TO COPING AND THE ENVIRONMENT?}

The fundamental defect in current cognitive approaches to depression is their utter lack of attention to the difficulties faced by depressed persons in their everyday environments, how they cope, and with what consequences. It is likely that future theorists and researchers will think it exceedingly odd that anyone attempted to explore and explain the cognitive processes of depressed persons without reference to these factors. The predictable result is a misattribution of distortion to the depressed persons' cognitive process of what is more properly seen as a feature of their exchange with the environment. Segal and Shaw offer little to correct this problem. In their survey, the depressed person's environment is seldom mentioned and has only a shadowy presence. Yet we remain hopeful that our earlier review will stimulate renewed interest in the possibility that the complaints of depressed persons have some basis in what is happening to them and, further, that the verbalizations of these complaints may be in part a miscarried effort to deal with these difficulties.

Some recent research has demonstrated how upset people are with depressed persons with whom they live, and it has been suggested further, "In light of ... [this], the emphasis of cognitive model on the depressed person's distorted thinking processes may be unnecessary or at least overstated, and great attention to the social context of depression may be warranted" (Coyne et al., in press, p. 17). Depressed persons deal with distressing circumstances that often do not yield to their efforts, and they often do so in the face of overtly hostile, critical, and rejecting significant others. It would seem that negative verbalizations about the self in such a context does not require the postulation of intractible cognitive processes. The modal depressed patient is probably a women with marital difficulties, and glib attempts to 
reduce her problems to a matter of distorted cognitions have potentially pernicious social implications. Yet that is what the current cognitive models of depressed seem to offer.

\section{SEGAL AND SHAW'S REAPPRAISAL REAPPRAISED}

The marks of a vigorous line of scientific inquiry include a clear link between methods and inference, serious considerations of alternative hypotheses, and the conduct of crucial experiments that have the potential for assisting a choice among these hypotheses (Platt, 1964). Segal and Shaw's implicit suggestions for the direction of future research do not meet such criteria, and they will be of little help in many attempts to resolve the pressing empirical, conceptual, and methodological challenges facing the cognitive perspective. Basically, they are reassuring the reader that (a) depressed-nondepressed differences on various measures can be accepted as prima facie evidence of depressed persons" "negative distorted thinking," (b) that current efforts to assess vulnerability to depression using the DAS are proving fruitful, and (c) that theorists and researchers can continue to ignore the environment.

If space permitted, we could identify other specific points of disagreement with Segal and Shaw. In lieu of that, we simply will state that in our judgment the role of cognition in depression and how to study it needs to be rethought in some fundamental ways. If further contributions to knowledge are to result, the study of cognition in depression has to get beyond the DAS, the ASQ, and simplistic interpretations of what are basically correlations between measures of depression and instruments tapping more specific aspects of the disorder. If accepted, the loosened standards and misplaced optimism displayed by Segal and Shaw can only diminish the future prospects of the cognitive perspective.

\section{REFERENCES}

Abramson, L. Y., Seligman, M. E. P., \& Teasdale, J. D. (1978). Learned helplessness in humans: Critique of reformulation. Journal of Abnormal Psychology, 87, 49-74.

Beck, A. T. (1967). Depression: Clinical, experimental and theoretical aspects. New York: Harper \& Row.

Beck, A. T. (1984). Cognition and therapy. Archives of General Psychiatry, 41, 1112-1114. Coyne, J. C., \& Gotlib, I. H. (1983). The role of cognition in depression: A critical appraisal. Psychological Bulletin, 94, 472-505.

Coyne, J. C., Kessler, R. C., Tal, M. Turnbull, J., Wortman, C., \& Greden, J. (in press). Living with a depressed person. Journal of Consulting and Clinical Psychology.

Eaves, E., \& Rush, A. J. (1984). Cognitive patterns in symptomatic and remitted unipolar depression. Journal of Abnormal Psychology, 93, 31-40. 
Hamilton, E. W., \& Abramson, L. Y. (1983). Cognitive patterns and major depressive disorder: A longitudinal study in a hospital setting. Journal of Abnormal Psychology, 92, 173-184.

Heil, J. (1983). Perception and cognition. Berkeley: University of California Press.

Klein, D. F. (1974). Endogenomorphic depression. Archives of General Psychiatry, 31, 447-454.

Kovacs, M., \& Beck, A. T. (1978). Maladaptive cognitive structures in depression. American Journal of Psychiatry, 135, 525-533.

Lewinsohn, P. M., Steinmetz, J. L., Larsen, D. W., \& Franklin, J. (1981). Depression-related cognitions: Antecedents or consequence? Journal of Abnormal Psychology, 90, 213-219.

Metalsky, G. I., Abramson, L. Y., Seligman, M. E. P., Semmel, A., \& Peterson, C. (1982). Attributional styles and life events in the classroom: Vulnerability and invulnerability to depressive mood reactions. Journal of Personality and Social Psychology, 43, $612-617$.

Platt, J. (1964). Strong inference. Science, 146, 347-352.

Reda, M. A. (1984). Cognitive organization and antidepressants: Attitude modification during amitriptyline treatment in severely depressed individuals. In M. A. Reda \& M. J. Mahoney (Eds.), Cognitive psychotherapies (pp. 119-149). Cambridge, Massachusetts: Ballinger.

Riskind, J. H., \& Rholes, W. S. (1984). Cognitive accessibility and the capacity of cognitions to predict future depression: A theoretical note. Cognitive Therapy and Research, 8, 1-12.

Riskind, J. H., \& Steer, R. E. (1984). Do maladaptive attitudes "cause" depression?: Misconception of cognitive theroy. Archives of General Psychiatry, 41, 1111.

Rush, A. J., \& Giles, D. E. (1982). Cognitive therapy: Theory and research. In A. J. Rush (Eds.), Short-term psychotherapies for depression. New York: Guilford Press.

Sacco, W. P., \& Beck, A. T. (1985). Cognitive therapy depression. In E. Beckham \& W. R. Leber (Eds.), Handbook of depression: Treatment, assessment, and research (pp. 3-38). Homewood, illinois: Dorsey Press.

Shaw, B. F. (1979). The theoretical and empirical foundations of a cognitive model of depression. In P. Pliner, I. Spigel, \& K. Blankstein (Eds.), Perception of emotion in self and others. New York: Plenum.

Silverman, J. S., Silverman, J. A., \& Eardley, D. A. (1984a). Do maladaptive attitudes cause depression? Archives of General Psychiatry, 41, 28-30.

Silverman, J. S., Silverman, J. A., \& Eardley, D. A. (1984). Reply to Riskind and Steer. $A r$ chives of General Psychiatry, 4I, 1112.

Simons, A. D., Garfield, S. L., \& Murphy, G. E. (1984). The process of change in cognitive therapy and pharmacotherapy of depression: Changes in mood and cognition. Archives of General Psychiatry, 41, 45-51.

Weissman, A. N., \& Beck, A. T. (1978). Development and validation of the Dysfunctional Attitude Scale: A preliminary investigation. Paper presented at the meeting of the American Educational Research Association, Toronto, Canada.

Williams, J. M. G. (1985). Attributional formulation of depression as a diathesis-stress model: Metalsky et al. reconsidered. Journal of Personality and Social Psychology, 48, 1572-1575. 